\title{
Revisión / Review \\ Essential oils of Cordia species, compounds and applications: a systematic review
}

[Aceites esenciales de especies de Cordia, compuestos y aplicaciones: una revisión sistemática]

\begin{abstract}
Kellen Cruvinel Rodrigues Andrade ${ }^{1}$, Diegue Henrique Nascimento Martins ${ }^{2}$, Diogo de Amorim Barros ${ }^{1}$, Paula Monteiro de Souza ${ }^{1}$, Dâmaris Silveira ${ }^{1}$, Yris Maria Fonseca-Bazzo ${ }^{1}$ \& Pérola de Oliveira Magalhães ${ }^{1}$
\end{abstract}

\author{
${ }^{1}$ Laboratory of Natural Products, Faculty of Health Science, University of Brasilia, Brasilia, Brazil \\ ${ }^{2}$ Department of Pharmacy, União Pioneira de Integração Social - UPIS, Campus II - Planaltina, Brazil.
}

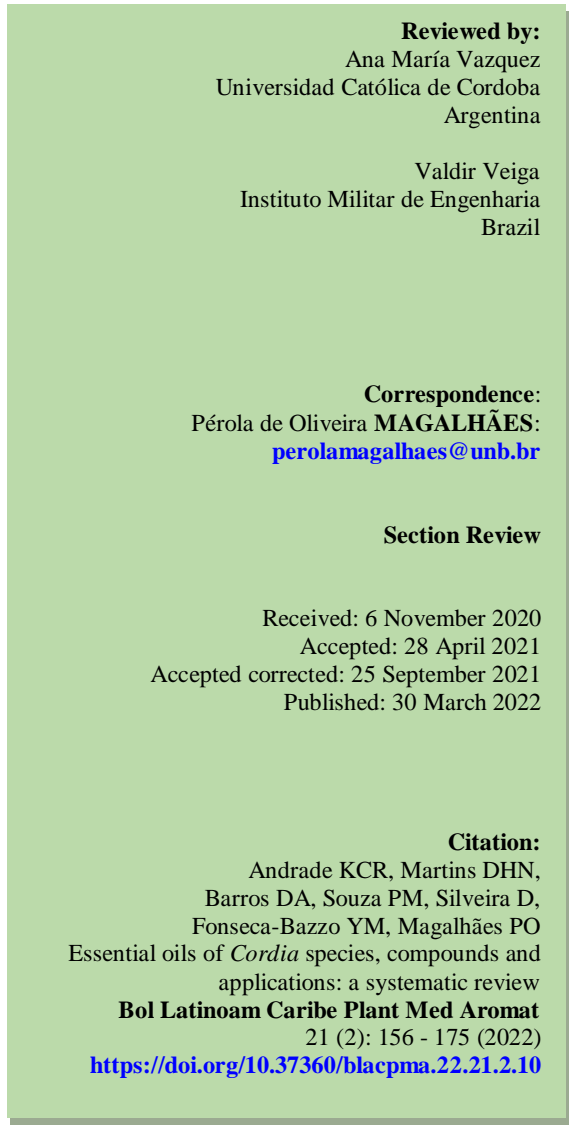

Abstract: The purpose of this systematic review was to identify the available literature on the essential oil from species of genus Cordia. This study followed the Preferred Reporting Items for Systematic Reviews. The search was conducted on four databases: LILACS, PubMed, Science Direct, and Scopus until June 5th, 2020, with no time or language restrictions. Sixty out of the 1,333 initially gathered studies fit the inclusion criteria after the selection process. Nine species of Cordia were reported in the selected studies, out of which $79 \%$ of the evaluated studies reported essential oil from Cordia curassavica. The essential oil extraction methods identified were hydrodistillation and steam distillation. As for biological application, antimicrobial, anti-inflammatory, larvicidal and antioxidant activities were the most reported. The main compounds reported for essential oil were $\beta$-caryophyllene, $\alpha$-humulene, $\alpha$-pinene, bicyclogermacrene, and sabinene. The information reported in this systematic review can contribute scientifically to the recognition of the importance of the genus Cordia.

Keywords: Cordia; Systematic review; Essential oil; Biological activity; Phytoconstituents.

Resumen: El propósito de esta revisión sistemática fue identificar la literatura disponible sobre el aceite esencial de especies del género Cordia. Este estudio siguió los elementos de informe preferidos para revisiones sistemáticas. La búsqueda se realizó en cuatro bases de datos: LILACS, PubMed, Science Direct y Scopus hasta el 5 de junio de 2020, sin restricciones de tiempo ni de idioma. Sesenta de los 1.333 estudios reunidos inicialmente cumplieron los criterios de inclusión después del proceso de selección. Se informaron nueve especies de Cordia en los estudios seleccionados, de los cuales el 79\% de los estudios evaluados informaron aceite esencial de Cordia curassavica. Los métodos de extracción de aceite esencial identificados fueron la hidrodestilación y la destilación al vapor. En cuanto a la aplicación biológica, las actividades antimicrobianas, antiinflamatorias, larvicidas y antioxidantes fueron las más reportadas. Los principales compuestos reportados para el aceite esencial fueron $\beta$-cariofileno, $\alpha$ humuleno, $\alpha$-pineno, biciclogermacreno y sabineno. La información reportada en esta revisión sistemática puede contribuir científicamente al reconocimiento de la importancia del género Cordia.

Palabras clave: Cordia; Revisión sistemática; Aceite esencial; Actividad biológica; Fitoconstituyentes. 


\section{INTRODUCTION}

The genus Cordia L. belongs to the Boraginaceae Juss. family, and presents four synonyms (Auxemma Miers, Gerascanthus P. Browne, Patagonula L., and Saccellium Humb. \& Bonpl.). They are found as shrubs, undergrowth, and trees. It is a non-endemic genus from Brazil but has confirmed occurrences in several Brazilian regions (North, Northeast, Midwest, Southeast, and South) (Flora do Brasil, 2019). The phytogeographic domains in Brazil for Cordia species are Amazon, Caatinga, Cerrado, Atlantic Forest, Pampa, and Pantanal. The vegetation types in which Cordia species can be found are Anthropic area, Caatinga (stricto sensu), Cerrado (lato sensu), Riparian Forest or Gallery, Lowland forest, Deciduous seasonal forest, Semideciduous seasonal forest, Ombrophilous forest (Rainforest), and Mixed ombrophilous forest (Flora do Brasil, 2019). Moreover, they occur from Central America down to the central region of Argentina (Matias et al., 2016). According to World Flora online Consortium (WFL, 2020), there are about 409 Cordia species and some of them are described as endangered.

The extract and the essential oil of some species of genus Cordia have been widely used as analgesic, anthelmintic, antiandrogenic activity, antiarthritic, antibacterial, antifertility, antifungal, antihistaminic, anti-HIV, anti-inflammatory, antimicrobial, antiserum action, anti-ulcer, antiviral, biliary obstruction, demulcent, hepatotoxic, juvenomimetic, larvicidal, leishmanicidal, nutritional food, spasmolytic, vasodilator, wound healing according to Thirupathi et al. (2008) and Matias et al. (2015). Several researches have carried out phytochemical studies resulting in the identification of different classes of secondary metabolites, as well the isolation of various constituents of different parts (root, stem, leaves, flowers and fruits) of various species of the genus Cordia, such as phenolic acid (e.g., rosmarinic acid), alkaloid (pyrrolizidine type), benzofurans, carotenoids (cerebroside), steroids ( $\beta$ sitosterol), flavonoids (7-methoxy flavone, 5,7,3',4'tetrahydroxy methoxyflavone, toxifolin-3, 5dirhamnoside, flavanols-artemetin, hesperetin-7rhamnoside, quercetin, quercitrin, rutin, toxifolin-3, quercetin-3-o-rutinoside), glycosides (dhurrin) fatty acids (linoleic, oleic, $\gamma$-linolinic, $\alpha$-linolinic acids), terpenes (tricyclene, alloaromadendrene, epi- $\alpha$ muurolol, trans-cariophyllene, $\alpha$-pinene, $\alpha$-muurolol, $\alpha$-cadinol, cordialin A, cordialin B), mucilage, fixed oils, polyphenol salts (calcium rosmarinate, cordigone, magnesium lithospermate, magnesium rosmarinate), suggars (arabinoglucan, D-glucose, Larabinose), saponin (lupan-20, 29-ene-3-O- $\beta$-Dmaltoside) and phenolic terpene (alliodorin) (Thirupathi et al., 2008).

Essential (or volatile) oils have characteristic fragrances and these metabolites may be extracted by steam or hydrodistillation, resulting in complex mixtures of different classes of known or unknown compounds as hydrocarbons, terpene alcohols, aldehydes, ketones, phenols, and esters (Adeosun et al., 2013; Alves et al., 2015). Essential oils may include activities such as antimicrobial agent, antioxidant, anti-inflamatory, anti-proliferative (Kumar et al., 2019; Kurti et al., 2019), antifungal, antinociceptive (Lasram et al., 2019), anti-tumor (Saldanha et al., 2019) and pesticidal (Wei et al., 2019). In addition to these activities, volatile oils are also used in the manufacture of perfumes, aromatic soaps, scented lotions, and cosmetics (Adeosun et al., 2013). In 2005, the first phytotherapy antiinflammatory drug of topical use in Brazil was developed from essential oil of $C$. curassavica. This phytotherapy is indicated for the topical treatment of tendonitis and muscular pain and the pharmacological studies have shown that the anti-inflammatory effects of the essential oil of $C$. curassavica can be attributed to two sesquiterpenes, $\alpha$-humulene and caryophyllene.

Given this broad approach to use and applications, the purpose of this systematic review was to identify the available literature referring to Cordia essential oil. These findings can guide researches towards a new perspective with Cordia essential oils.

\section{METHODS}

This systematic review was conducted following the PRISMA (Preferred Reporting Items for Systematic Reviews and Meta-Analysis) Checklist (Moher et al., 2009). The protocol was not registered because it is a systematic review of in vitro studies. This type of systematic review is not eligible for inclusion in the International Prospective Register of Systematic Reviews (PROSPERO).

\section{Eligibility criteria: Inclusion criteria}

Articles that focused on studies with essential oil of Cordia species, evaluating the method of extraction, 
biological activity, and the phytocomposition were eligible for inclusion.

\section{Exclusion criteria}

The following studies were excluded: (1) Papers did not report about essential oil of Cordia; (2) Reviews, letters, personal opinions, book chapters, and conference abstracts.

\section{Study selection}

The study selection was completed in two phases. In phase one, two authors independently reviewed the titles and abstracts of all the references. These authors selected studies that appeared to meet the inclusion criteria based on their titles and abstracts. A third author was consulted when disagreements emerged between the two initial evaluators. Any studies that did not fulfill the inclusion criteria were discarded. In phase two, two authors read all the fulltext articles and excluded those which were not in agreement with the inclusion criteria. The authors independently reviewed all full-text articles. Any disagreement in either phase was resolved by discussion and mutual agreement among the reviewers.

\section{Information sources and search strategy}

Detailed individual search strategies for each of the following bibliographic databases were developed: LILACS, PubMed, Science Direct, and Scopus (Appendix B).

A partial gray literature search was performed using Google Scholar. The search included all articles published up only until June $5^{\text {th }}$, 2020 , across all databases with no time or language restrictions. Besides, the reference lists of selected articles were carefully checked for potentially relevant studies that could have been missed during the electronic database search. Duplicated references were removed using reference manager software (EndNote X6, Thomson Reuters).

\section{Data collection process and data items}

Two authors collected the required information from the selected articles. A third author independently checked the data extraction for accuracy and detail. Again, any disagreement in either phase was resolved by discussion and mutual agreement among the authors. For all the included studies, the following information was recorded: author(s), year of publication, species of Cordia, part of the plant, essential oil extraction methods, and biological activity. The authors independently reviewed all fulltext articles.

\section{Summary measures}

Cordia species producing, part of the plant, extract method, biological activity, and isolated compound of essential oil were the main evaluated outcomes.

\section{RESULTS}

\section{Study selection}

In the phase 1 of study selection, 1,333 citations were identified across the four electronic databases: LILACS, PubMed, Science Direct, and Scopus. After the duplicate articles were removed, remained 1032 citations. Comprehensive evaluation of the abstracts was completed and 957 articles were excluded, so 75 articles remained after phase 1. Forty-nine articles were identified using Google Scholar, but one were included. A full-text review was conducted on these 76 articles. This process led to the exclusion of 16 papers (Appendix C). Thus, 60 articles were selected after the identification, screening, inclusion, and exclusion of studies used for this systematic review (Figure No. 1).

\section{Study characteristics}

A summary of the descriptive characteristics of the included studies is provided in Table No. 1. The studies were carried out in different countries, published between 1990 and 2020, and were in English or Portuguese. The highest frequency of publication was observed from 2007. All the selected studies were conducted addressing Cordia essential oil. Most studies have addressed the biological activity and/or phytoconstituents identification of Cordia essential oil. Sixty-three articles were selected, but five dealing with essential oil of Cordia did not have enough information on biological activity, phytochemicals, and plant drugs used in the extraction process: pharmacokinetics and tissue distribution of phytoconstituents of essential oil (Chaves et al., 2008); methodology for quantifying $C$. curassavica in raw materials and pharmaceutical formulations (Gomes et al., 2019); histopathological, immunohistochemical and biochemical of essential oil topical application (Perini et al., 2015); clinical evaluation of the efficacy and safety of the use of $C$. curassavica essential oil (Refsio et al., 2005); release 
and permeation in vitro of $C$. curassavica essential oil from topical forms (Silva et al., 2019c) and secretion of essential oil in C. curassavica glandular trichomes (Ventrella \& Marinho, 2008).

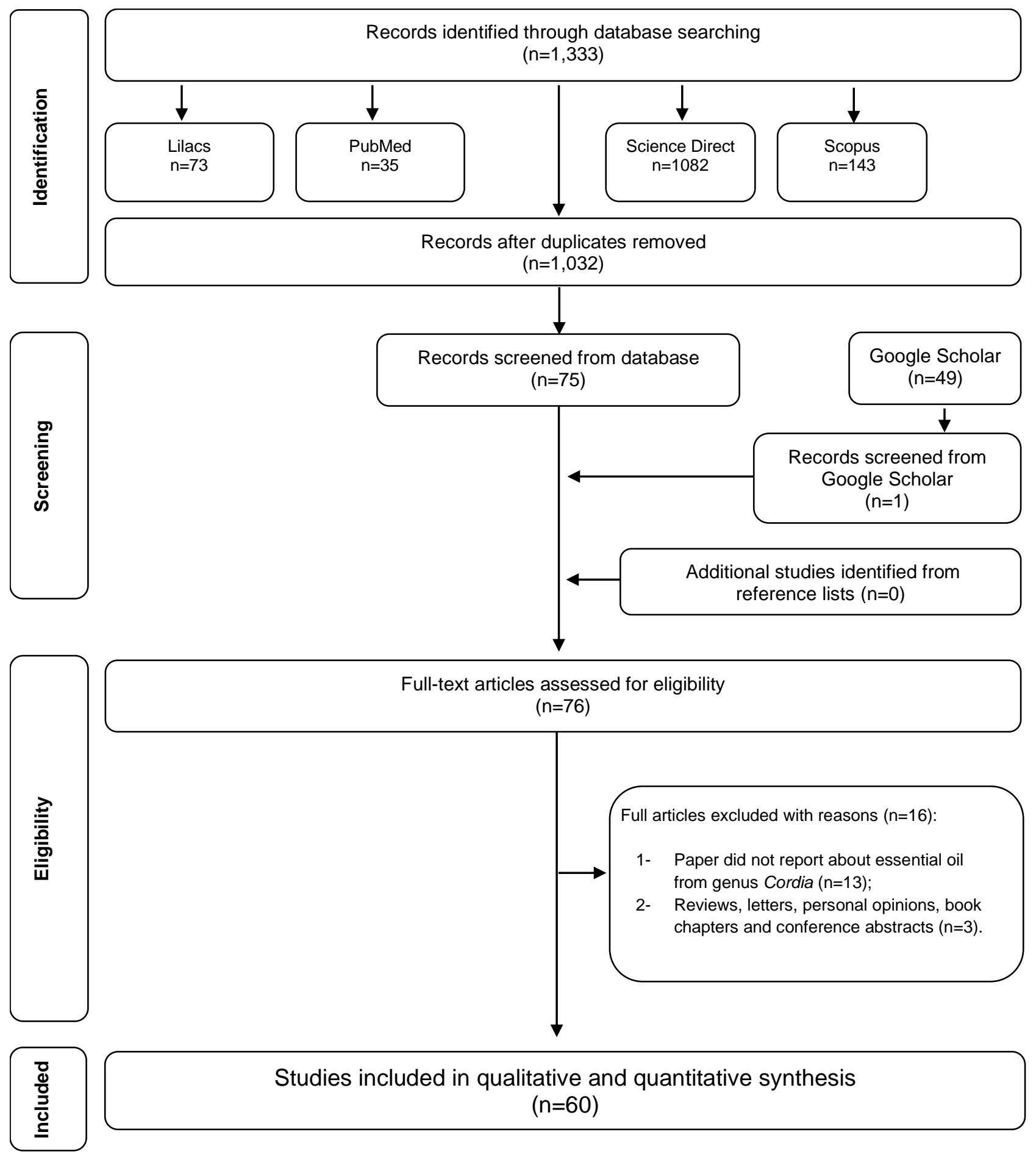

Figure No. 1

Flow Diagram of literature search and selection criteria for Cordia genus adapted from PRISMA

Boletín Latinoamericano y del Caribe de Plantas Medicinales y Aromáticas / 159 


\section{Synthesis of results: Cordia species and nomenclature}

The names of plant species of the genus Cordia were compared to the list belonging to The World Flora to verify the proper use of the respective name and synonym. Among the 60 articles evaluated in this review, twelve species were cited (Table No. 1), according to The World Flora (WFL, 2020).

The most cited species was $C$. curassavica (Jacq) Roemer \& Schultes (79\%), being clustered of the following names: $C$. verbenacea A. DC. (synonym) - 53\%; Varronia curassavica Jacq. (synonym) $-16.5 \%$; C. curassavica (Jacq) Roemer \& Schultes (official name) $-8 \%$ and Cordia chacoensis (synonym) $-1.5 \%$. The other citations were from $C$. globosa (Jacq.) H. B. K., Cordia sebestena (L.),
Cordia trichotoma (Vell.) Arráb. ex Steud. being 3\% for each, and Cordia africana Lam., Cordia cylindrostachya Roem. \& Schult., Cordia gilletii, Cordia leucocephala Moric, Cordia leucomalloides, Cordia multispicata Cham., Cordia myxa L., Cordia nitida Vahl. being $1.5 \%$ for each.

Compared to The World Flora database, only 4 studies (V. curassavica Jacq.; C. trichotoma (Vell.) Arráb. ex Steud.; $C$. leucocephala Moric e $C$. multispicata Cham.) used the nomenclature consistent with this database. The other citations presented divergence regarding writing. Therefore, it was decided to standardize all the species reported in those systematic review of articles following the nomenclature presented by The World Flora Online Consortium (WFL, 2020).

Table No. 1

Summary of descriptive characteristics of included studies $(\mathrm{n}=60)$

\begin{tabular}{|c|c|c|c|c|c|}
\hline Species & Extract method & $\begin{array}{l}\text { Part of } \\
\text { the } \\
\text { plant }\end{array}$ & Biolocial activity & Phytoconstituents & Author \\
\hline Cordia africana Lam. & Hydrodistillation & Leaves & Cytotoxicity & $\begin{array}{l}\beta \text {-caryophyllene } \\
\text { germacrene D } \\
\delta \text {-cadinene } \\
\text { phytol }\end{array}$ & Ashmawy et al., 2020 \\
\hline Cordia chacoensis & Steam distillation & $\begin{array}{l}\text { Aerial } \\
\text { parts }\end{array}$ & - & $\begin{array}{l}6 \alpha \text {-hydroxygermacra- } \\
1(10), 4 \text {-diene } \\
\beta \text {-caryophyllene }\end{array}$ & Arze et al., 2013 \\
\hline \multirow{8}{*}{$\begin{array}{l}\text { Cordia curassavica } \\
\text { (Jacq.) Roem. \& Schult. }\end{array}$} & Hydrodistillation & $\begin{array}{l}\text { Fresh } \\
\text { leaves }\end{array}$ & Pesticidal & $\begin{array}{l}\alpha \text {-santalene } \\
\beta \text {-sinensal } \\
(Z) \text { - } \alpha \text {-trans- } \\
\text { bergamotene }\end{array}$ & Alves et al., 2015 \\
\hline & Hydrodistillation & Leaves & Antibacterial & - & Ambrosio et al., 2017 \\
\hline & Hydrodistillation & $\begin{array}{l}\text { Aerial } \\
\text { parts }\end{array}$ & $\begin{array}{l}\text { Antibacterial and } \\
\text { antifungal }\end{array}$ & $\begin{array}{l}\alpha \text {-pinene } \\
\text { trans-caryophyllene } \\
\text { alloaromadendrene }\end{array}$ & Carvalho et al., 2004 \\
\hline & - & - & Anti-inflammatory & - & Carvalho \& Barja, 2012 \\
\hline & Hydrodistillation & Leaves & $\begin{array}{l}\text { Antibacterial and } \\
\text { bacterial resistance } \\
\text { modulating }\end{array}$ & $\begin{array}{l}\text { sabinene } \\
\beta \text {-caryophyllene } \\
\text { bicyclogermacrene } \\
\text { germacrene D } \\
\text { spathulenol }\end{array}$ & Carvalho et al., 2017 \\
\hline & - & - & - & $\alpha$-humulene & Chaves et al., 2008 \\
\hline & Hydrodistillation & Leaves & Antifungal & - & Duarte et al., 2005 \\
\hline & Hydrodistillation & Leaves & - & $\alpha$-humulene & Facanali et al., 2020 \\
\hline
\end{tabular}

Boletín Latinoamericano y del Caribe de Plantas Medicinales y Aromáticas / 160 


\begin{tabular}{|c|c|c|c|c|}
\hline Hydrodistillation & Leaves & - & - & Feijó et al., 2014 \\
\hline Hydrodistillation & $\begin{array}{l}\text { Aerial } \\
\text { parts }\end{array}$ & Anti-inflammatory & $\begin{array}{l}\alpha \text {-humulene } \\
\beta \text {-caryophyllene }\end{array}$ & Fernandes et al., 2007 \\
\hline- & $\begin{array}{l}\text { Stems } \\
\text { and } \\
\text { leaves }\end{array}$ & - & $\begin{array}{l}\alpha \text {-humulene } \\
\beta \text {-caryophyllene }\end{array}$ & Gomes et al., 2019 \\
\hline Hydrodistillation & Leaves & Food deterrent & $\begin{array}{l}\beta \text {-terpinene } \\
\text { sabinene } \\
\alpha \text {-pinene }\end{array}$ & Gómez et al., 1999 \\
\hline Hydrodistillation & Leaves & - & $\alpha$-bisabolol & Guimarães et al., 2015 \\
\hline Hydrodistillation & $\begin{array}{l}\text { Aerial } \\
\text { parts }\end{array}$ & $\begin{array}{l}\text { Antibacterial and } \\
\text { antifungal }\end{array}$ & $\begin{array}{l}\delta \text {-elemene } \\
\beta \text {-eudesmol } \\
\text { spathulenol } \\
\text { cadina } 4(5), 10(14) \\
\text { diene }\end{array}$ & Hernandez et al., 2007 \\
\hline Hydrodistillation & - & Antifungal & pulegone & Hoyos et al., 2012 \\
\hline- & Leaves & Anti-inflammatory & - & Leonardi et al., 2010 \\
\hline Hydrodistillation & Leaves & - & $\begin{array}{l}\beta \text {-elemene } \\
\beta \text {-caryophyllene } \\
\text { amorpha4,7(11)-diene, } \\
\text { eudesm-7 (11)-en-4- } \\
\text { ol, } \\
\alpha \text {-humulene } \\
\delta \text {-elemene } \\
\delta \text {-cadinene } \\
\text { 9-epi-(E)- } \\
\text { caryophyllene }\end{array}$ & Marques et al., 2019 \\
\hline Hydrodistillation & Leaves & $\begin{array}{l}\text { Antibacterial and } \\
\text { bacterial resistance } \\
\text { modulating }\end{array}$ & $\begin{array}{l}\text { sabinene } \\
\text { sabinene hydrate }\end{array}$ & Matias et al., 2016 \\
\hline Hydrodistillation & $\begin{array}{l}\text { Aerial } \\
\text { parts }\end{array}$ & Antibacterial & $\begin{array}{l}\text { tricyclene } \\
\text { bicyclogermacrene } \\
\text { germacrene D } \\
\beta \text {-caryophyllene }\end{array}$ & Meccia et al., 2009 \\
\hline- & - & Anti-inflammatory & $\begin{array}{l}\alpha \text {-humulene } \\
\beta \text {-caryophyllene }\end{array}$ & Medeiros et al., 2007 \\
\hline Hydrodistillation & Leaves & Antifungal & $\begin{array}{l}\text { tricyclene } \\
\text { camphene } \\
\beta \text {-caryophyllene } \\
\beta \text {-sesquiphellandrene } \\
\alpha \text {-zingiberene } \\
\text { 7-cyclodecen-1-one } \\
\text { 7-methyl-3- } \\
\text { methylene-10 (1) } \\
\text { propyl }\end{array}$ & Nizio et al., 2015 \\
\hline
\end{tabular}




\begin{tabular}{|c|c|c|c|c|}
\hline & & & turmerone & \\
\hline Hydrodistillation & Leaves & Antiprotozoal & $\begin{array}{l}\alpha \text {-pinene } \\
\text { epiglobulol } \\
\beta \text {-caryophyllene } \\
\text { sabinene }\end{array}$ & Nizio et al., 2018 \\
\hline & Leaves & - & $\begin{array}{l}\beta \text {-phellandrene } \\
\text { cubebol }\end{array}$ & \\
\hline Hydrodistillation & Stems & - & $\begin{array}{l}\text { spathulenol } \\
\text { trans-sesquisabinene } \\
\text { hydrate }\end{array}$ & Oliveira et al., 2007 \\
\hline Hydrodistillation & $\begin{array}{l}\text { Fresh } \\
\text { leaves }\end{array}$ & $\begin{array}{l}\text { Anti-inflammatory } \\
\text { and anti-allergic }\end{array}$ & $\begin{array}{l}\beta \text {-caryophyllene } \\
\alpha \text {-humulene }\end{array}$ & Passos et al., 2007 \\
\hline- & - & - & - & Perini et al., 2015 \\
\hline Hydrodistillation & Leaves & $\begin{array}{l}\text { Anti-inflammatory } \\
\text { and antibacterial }\end{array}$ & $\alpha$-humulene & Pimentel et al., 2012 \\
\hline Hydrodistillation & Leaves & - & $\begin{array}{l}\beta \text {-caryophyllene } \\
\gamma \text {-muurolene } \\
\text { elixene }\end{array}$ & Queiroz et al., 2016 \\
\hline- & - & Anti-inflammatory & - & Refsio et al., 2005 \\
\hline Hydrodistillation & Leaves & Anti-periodontitis & $\alpha$-humulene & Ribeiro et al., 2012 \\
\hline Hydrodistillation & $\begin{array}{l}\text { Fresh } \\
\text { leaves }\end{array}$ & $\begin{array}{l}\text { Antibacterial and } \\
\text { antifungal }\end{array}$ & $\begin{array}{l}\beta \text {-caryophyllene } \\
\text { bicyclogermacrene } \\
\delta \text {-cadinene } \\
\alpha \text {-pinene }\end{array}$ & Rodrigues et al., 2012 \\
\hline- & $\begin{array}{l}\text { Leaves } \\
\text { and } \\
\text { stems }\end{array}$ & Antibacterial & - & Sánchez et al., 2018 \\
\hline Hydrodistillation & Leaves & Larvicidal & $\begin{array}{l}\alpha \text {-pinene } \\
\beta \text {-pinene } \\
\beta \text {-caryophyllene } \\
\text { bicyclogermacrene }\end{array}$ & Santos et al., 2006 \\
\hline Hydrodistillation & Leaves & - & $\begin{array}{l}\beta \text {-elemene } \\
\alpha \text {-humulene } \\
\text { bisabolene } \\
\text { selin-11-en-4- } \alpha \text {-ol } \\
\text { trans- } \alpha \text {-trans- } \\
\text { bergamotol }\end{array}$ & Santos et al., 2013 \\
\hline Hydrodistillation & Leaves & - & $\begin{array}{l}\alpha \text {-pinene } \\
\alpha \text {-santalene } \\
\text { (E)- } \alpha \text {-santalal } \\
\text { (E) - } \alpha \text {-bergamotenal }\end{array}$ & Sciarrone et al., 2017 \\
\hline Hydrodistillation & $\begin{array}{l}\text { Aerial } \\
\text { parts }\end{array}$ & Antifungal & $\begin{array}{l}\alpha \text {-pinene } \\
\text { sabinene } \\
\text { limonene }\end{array}$ & Silva et al., 2012a \\
\hline
\end{tabular}

Boletín Latinoamericano y del Caribe de Plantas Medicinales y Aromáticas / 162 
$\beta$-phelandrene

1,8-cineol

$\alpha$-copaene

$\beta$-elemene

$\beta$-caryophyllene

$\alpha$-trans-bergamotene

$\alpha$-humulene

germacrene $\mathrm{D}$

bicyclogermacrene

spathulenol

caryophyllene oxide

epoxy-2-humulene

epi- $\alpha$-cadinol

turmerone

curlone

methyl farnesate

2E, 6Z-farnesol

2E, 6E-farnesol

methyl 2E, $6 \mathrm{E}$

farnesoate

methyl 2E, 6E

farnesoate epoxide

\begin{tabular}{|c|c|c|c|c|}
\hline Hydrodistillation & $\begin{array}{l}\text { Aerial } \\
\text { parts }\end{array}$ & Antifungal & - & Silva et al., 2012b \\
\hline Hydrodistillation & $\begin{array}{l}\text { Leaves } \\
\text { and } \\
\text { flowers }\end{array}$ & Antifungal & - & Silva et al., 2014a \\
\hline Hydrodistillation & $\begin{array}{l}\text { Aerial } \\
\text { parts }\end{array}$ & Antifungal & $\begin{array}{l}\beta \text {-caryophyllene } \\
\text { methyl } 2 \mathrm{E}, 6 \mathrm{E} \\
\text { farnesoate }\end{array}$ & Silva et al., 2014b \\
\hline Hydrodistillation & Leaves & $\begin{array}{l}\text { Antibacterial and } \\
\text { antifungal }\end{array}$ & $\begin{array}{l}\alpha \text {-pinene } \\
\text { sabinene } \\
\beta \text {-caryophyllene } \\
\text { ar-curcumene } \\
\beta \text {-sesquiphellandrene } \\
\text { 4-cyclodecen-1-one } \\
\text { ar-turmerone }\end{array}$ & Silva et al., 2020 \\
\hline Hydrodistillation & Leaves & Antiprotozoal & $\begin{array}{l}\alpha \text {-pinene } \\
\text { 7-cyclodecen-1-one,7- } \\
\text { methyl-3-methylene- } \\
\text { 10-(1-propyl) } \\
\text { germacrene-D-4-ol } \\
\text { ar-curcumene } \\
\beta \text {-caryophyllene } \\
\text { ar-turmerone }\end{array}$ & Silva et al., 2019a \\
\hline & - & - & - & Silva et al., 2019b \\
\hline
\end{tabular}

Boletín Latinoamericano y del Caribe de Plantas Medicinales y Aromáticas / 163 


\begin{tabular}{|c|c|c|c|c|}
\hline- & Leaves & - & - & Ventrella et al., 2008 \\
\hline Hydrodistillation & Leaves & - & $\begin{array}{l}\alpha \text {-pinene } \\
\beta \text {-pinene } \\
1,8 \text {-cineol } \\
\beta \text {-elemene } \\
\beta \text {-caryophyllene } \\
\alpha \text {-humulene } \\
\beta \text {-santalene } \\
\text { germagrene-D } \\
\beta \text {-bisadolene } \\
\delta \text {-cadinene } \\
\text { caryophyllene oxide } \\
(Z) \text { - } \alpha \text {-trans- } \\
\text { bergamotol acetate } \\
(Z) \text {-epi- } \beta \text {-santalol }\end{array}$ & Zotti-Sperotto et al., 2020 \\
\hline Steam distillation & $\begin{array}{l}\text { Aerial } \\
\text { parts }\end{array}$ & $\begin{array}{l}\text { Antibacterial and } \\
\text { antifungal }\end{array}$ & $\begin{array}{l}\text { germacrene } \\
\text { tricyclene } \\
\alpha \text {-pinene } \\
\text { isocariophyllene } \\
\text { selinene } \\
\text { D-limonene }\end{array}$ & Hernández et al., 2014 \\
\hline Steam distillation & Leaves & $\begin{array}{l}\text { Antinociception } \\
\text { and anti- } \\
\text { inflammatory }\end{array}$ & $\begin{array}{l}\alpha \text {-humulene } \\
\text { geranylgeraniol } \\
\beta \text {-caryophyllene }\end{array}$ & Basting et al., 2019 \\
\hline Steam distillation & Leaves & Acaricidal & $\begin{array}{l}\alpha \text {-thujene } \\
\alpha \text {-pinene } \\
\text { sabinene } \\
\beta \text {-pinene } \\
\beta \text {-myrcene } \\
\text { limonene } \\
1,8 \text {-cineol } \\
\text { bornyl acetate } \\
\text { citronellyl acetate } \\
\delta \text {-elemene } \\
\alpha \text {-copaene } \\
\beta \text {-boubornene } \\
\beta \text {-elemene } \\
\text { sesquitujene } \\
\alpha \text {-cis-bergamotene } \\
\beta \text {-caryophyllene } \\
\beta \text {-gurgujene } \\
\alpha \text {-trans-bergamotene } \\
\text { cis- } \beta \text {-farnesene }\end{array}$ & Castro et al., 2019 \\
\hline
\end{tabular}

Boletín Latinoamericano y del Caribe de Plantas Medicinales y Aromáticas / 164 


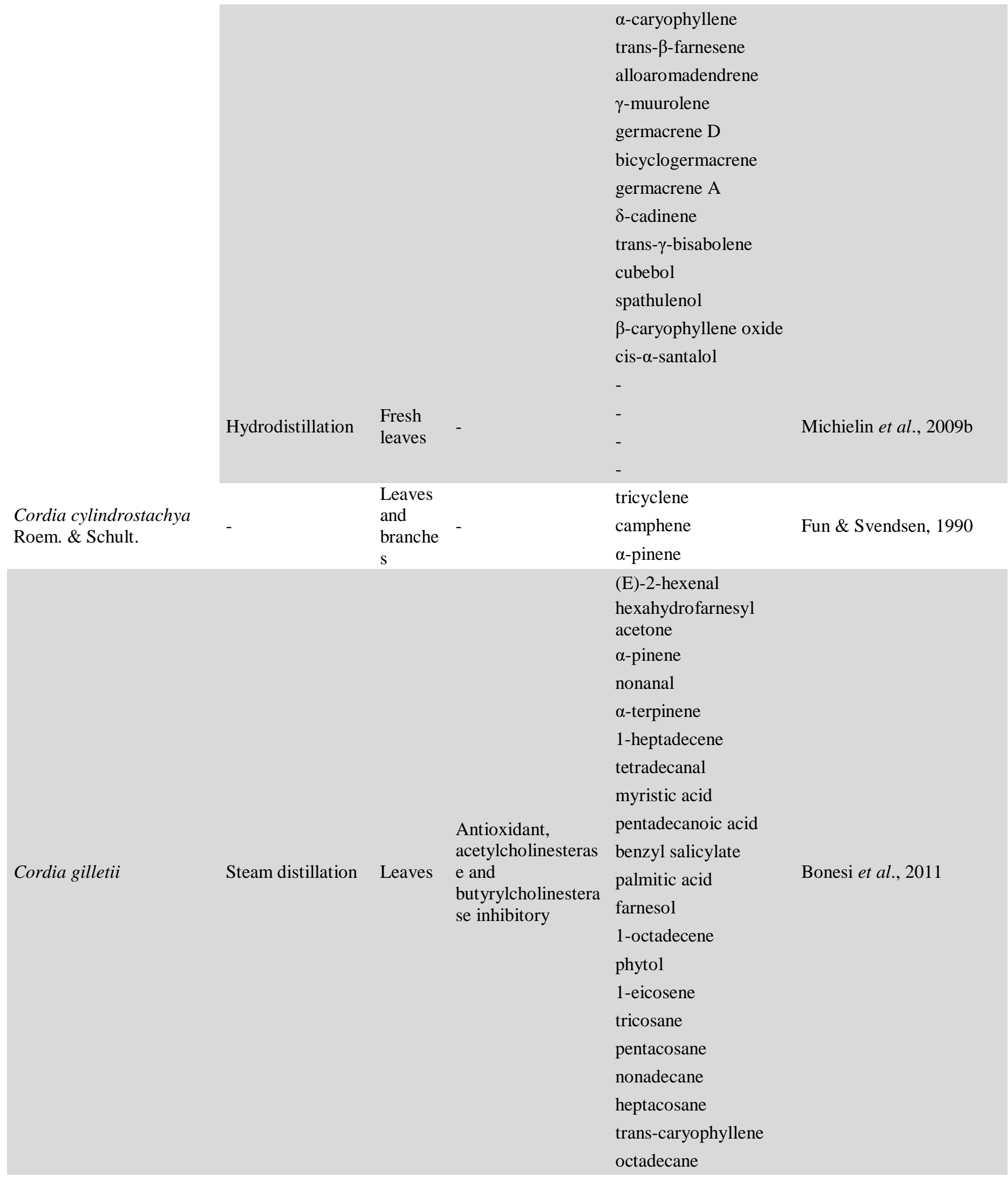




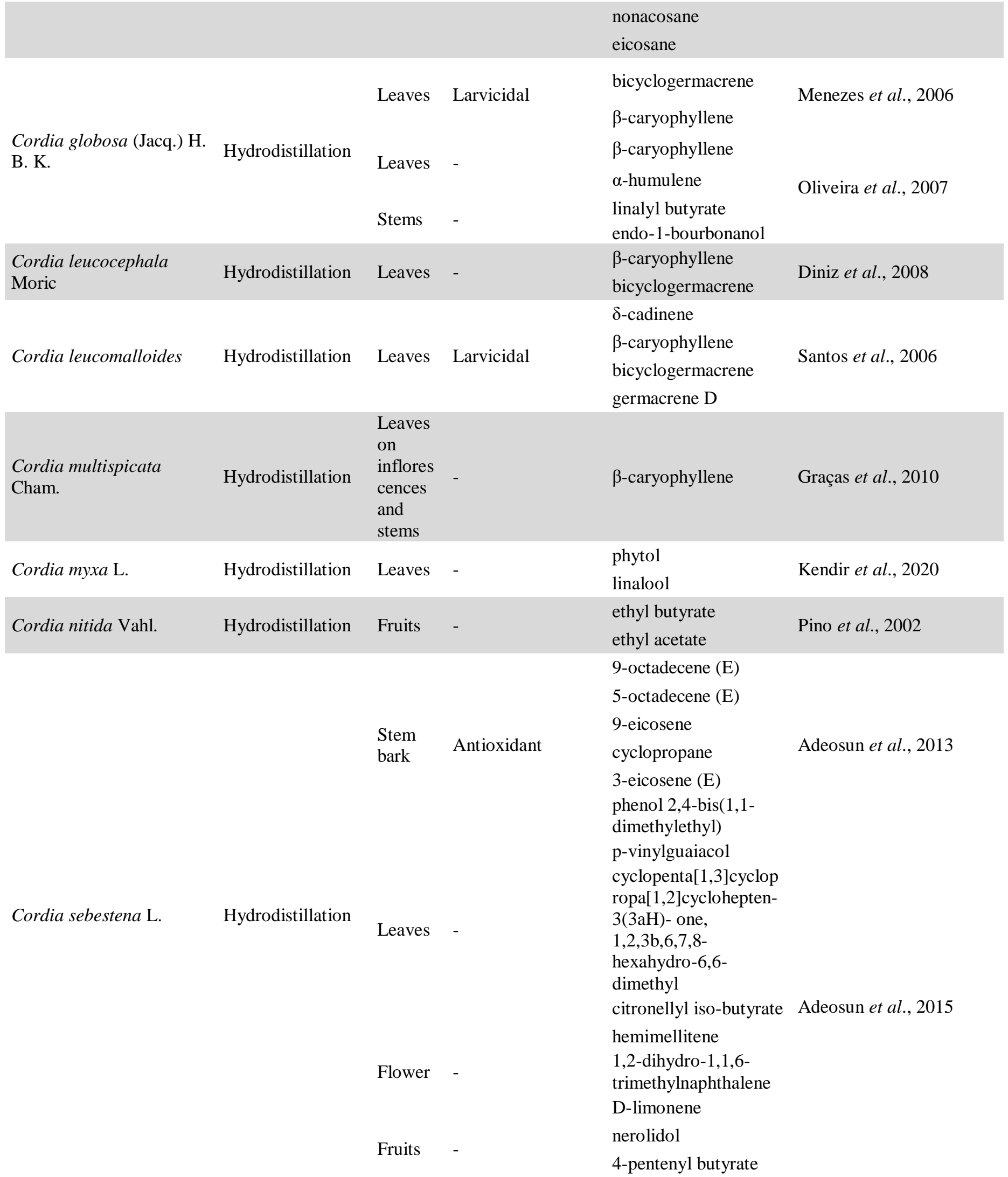




\begin{tabular}{|c|c|c|c|c|}
\hline \multirow{3}{*}{$\begin{array}{l}\text { Cordia trichotoma (Vell.) } \\
\text { Arráb. ex Steud. }\end{array}$} & & & farnesyl acetate & \\
\hline & Hydrodistillation & $\begin{array}{l}\text { Heartw } \\
\text { ood and } \\
\text { sapwoo } \\
\text { d }\end{array}$ & $\begin{array}{l}\alpha \text {-cadinol } \\
\alpha \text {-muurolol } \\
\text { epi- } \alpha \text {-muurolol } \\
\delta \text {-cadinene } \\
\text { guaia } 3,10 \text { (4) -dien- } \\
11 \text {-ol }\end{array}$ & Menezes et al., 2005 \\
\hline & $\begin{array}{l}\text { Steam Drag } \\
\text { Distillation }\end{array}$ & Wood & $\begin{array}{l}\alpha \text {-muurolol } \\
\alpha \text {-cadinol }\end{array}$ & Wille et al., 2017 \\
\hline
\end{tabular}

\section{Essential oil extraction methods}

Among the methods used for extraction of essential oil from Cordia species, were hydrodistillation (73\%) and stem distillation (18\%). On the other hand, $9 \%$ of the articles did not report the method of essential oil extraction.

Extraction of essential oils performed by hydrodistillation indicated that most of these studies $(69 \%)$ used the leaves, and among them, 9\% referred to fresh leaves. Other plant parts were also used, such as aerial parts $(17 \%)$, fruits $(2 \%)$, flowers $(2 \%)$, stem $(2 \%)$, stem bark (2\%), heartwood (2\%), and sapwood (2\%). Among the consulted articles, $2 \%$ did not report the parts of the plants that were used in the hydrodistillation process.

\section{Biological activity}

Among the evaluated articles, the highest citation for biological activity for Cordia species was antimicrobial activity (30\%), meaning antibacterial $(16 \%)$ and antifungal (14\%). In addition to this activity, the following activities were also observed: anti-inflammatory (10\%), larvicidal (4\%), antioxidant (3\%), bacterial resistance modulating (3\%), antiprotozoal (3\%), acaricidal (1\%), anti-allergic $(1 \%)$, antinociception (1\%), acetylcholinesterase inhibitory $(1 \%)$, butyrylcholinesterase inhibitory (1\%), anti-periodontitis (1\%), cytotoxicity (1\%), food deterrent $(1 \%)$ and pesticidal (1\%). From the evaluated articles, it was identified that $38 \%$ of the studies did not report biological activities for Cordia species.

The essential oil and extract of Cordia species have been widely used as antimicrobial and anti-inflammatory. Although many researchers have reported anti-inflammatory activity of the essential oil of C. curassavica, only 8 studies (13\%) showed the anti-inflammatory effect found in the leaves of the plant.

Among the studies on antibacterial activity (16\%), all of them referred to $C$. curassavica. The extraction methods for essential oils were hydrodistillation $(84.6 \%)$ and steam distillation (7.7\%); $7.7 \%$ did not report the extraction method. In the hydrodistillation process, the plant parts used were leaves $(73 \%)$ and aerial parts $(27 \%)$. In the essential oil extraction process by steam distillation was used the aerial parts.

In the same way, studies showing antifungal activity (14\%) were found only for C. curassavica. The extraction methods were hydrodistillation $(91 \%)$ and steam distillation (9\%). For the hydrodistillation process, the plant parts were aerial parts $(50 \%)$, leaves $(34 \%)$, flowers $(8 \%)$, and one work did not report the plant part $(8 \%)$. By the steam distillation method, the extraction was from aerial parts.

In general, the tested Gram-positive bacteria and fungi were sensitive to the essential oil of $C$. curassavica, but most of the Gram-negative bacteria were resistant. A screening works on the antimicrobial activity of essential oil of $C$. curassavica, showed Bacillus cereus was the most sensitive (100\%) species, followed by Staphylococcus aureus (75\%) and Staphylococcus epidermidis $(67 \%)$ of the time-sensitive when tested. The genus Candida was the most used (52\%) to verify the antifungal activity of C. curassavica essential oil.

Most of the antibacterial activity evaluation referred to the disk diffusion method (43\%) and by minimum inhibitory concentration (MIC) (36\%). Also, minimal bactericidal concentration (MBC) $(14 \%)$ or identification by polymerase chain reaction (PCR) (7\%) were cited. 
For the evaluation of antifungal activity, it was used, mainly, the observation of the compartment of inhibition of essential oil in the growth of the fungus in culture medium (50\%), followed by disk diffusion method (25\%) and MIC $(25 \%)$.

\section{Phytoconstituents}

A total of 106 compounds were found through the performed research. These constituents were grouped according to their chemical structures, and the presence of terpenes (55\%), hydrocarbons (42\%), and esters $(3 \%)$ was noted. Among the terpenes, $72 \%$ of phytoconstituents were sesquiterpenes, and $28 \%$ were monoterpenes.Among the sesquiterpenes, the most reported were $\beta$-caryophyllene $(25.9 \%), \alpha$-humulene $(9 \%)$, bicyclogermacrene $(8 \%)$, germacrene D $(7 \%)$, $\beta$-elemene $(4.5 \%)$, spathulenol $(3.5 \%), \delta$-cadinene (3.5\%), $\gamma$-muurolene $(1.9 \%), \alpha$-cadinol $(1.9 \%), \alpha$ copaene $(1.9 \%), \alpha$-muurolol $(1.9 \%), \alpha$-santalene
(1.9\%), $\alpha$-trans-bergamotene (1.9\%), cubebol (1.9\%), epi- $\alpha$-cadinol (1.9\%), germacrene A $(1.9 \%), \beta$ sesquiphellandrene $(1.9 \%), \delta$-elemene $(1.9 \%), \beta$ eudesmol (1.9\%). While among the monoterpenes the most reported were: $\alpha$-pinene $(34 \%)$, sabinene $(16 \%)$, limonene (9\%), $\beta$-pinene (7\%), 1,8-cineol (7\%), $\beta$ phellandrene $(4 \%)$, camphene $(4 \%)$, caryophyllene oxide $(4 \%)$.

The most cited hydrocarbons were phytol (7\%) and $\beta$-sinensal (5\%). The esters reported were methyl 2E,6E-farnesoate (40\%), 4-pentenyl butyrate $(40 \%)$ and benzyl salicylate $(20 \%)$.

Then, of this survey of phytoconstituents in the characterization of essential oil, the most reported were $\beta$-caryophyllene, $\alpha$-humulene, $\alpha$-pinene, bicyclogermacrene, and sabinene. The chemical structures of these phytoconstituents are illustrated in Figure No. 2. Among the 60 articles reviewed in this study, $22 \%$ not report the phytoconstituents.

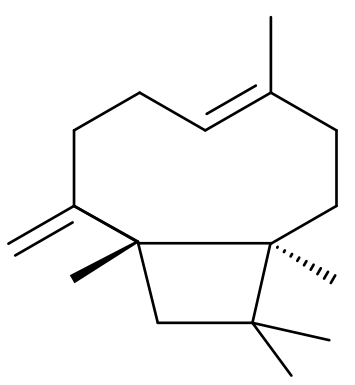

(1)

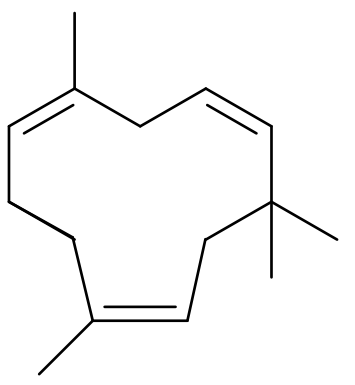

(2)

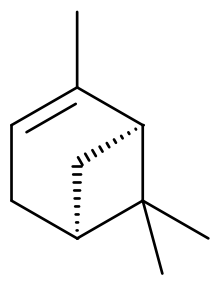

(3)

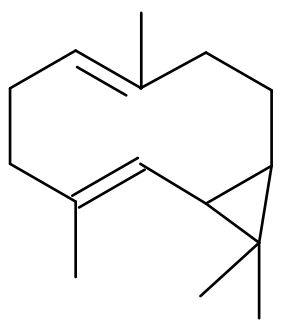

(4)

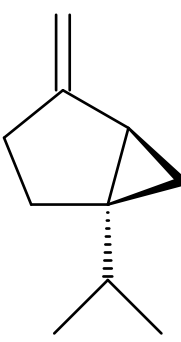

(5)

Figure No. 2

Chemical structures of most the cited phytoconstituents in essential oil of Cordia. (1) $\beta$-caryophyllene, (2) $\alpha$ humulene, (3) $\alpha$-pinene, (4) bicyclogermacrene, and (5) sabinene

\section{Preclinical evaluation}

Among the 60 studies, nine (15\%) addressed the preclinical evaluation of Cordia essential oil. From these evaluations, the only species studied was $C$. curassavica. As for biological activity evaluated in the preclinical stage were anti-inflammatory $(64 \%)$, antinociception (9\%), anti-allergic (9\%), antiprotozoal (9\%) and anti-periodontitis $(9 \%)$.

Fernandes et al. (2007) evaluated the anti- inflammatory properties of $\alpha$-humulene and $\beta$ caryophyllene isolated from $C$. curassavica essential oil. In results revealed that oral treatment with both compounds displayed marked inhibitory effects in different inflammatory experimental models in mice and rats. The same group after, evaluated the effects of these compounds on the acute inflammatory responses elicited by LPS in the rat paw and observed the effect of preventing LPS-induced inflammation

Boletín Latinoamericano y del Caribe de Plantas Medicinales y Aromáticas / 168 
(Medeiros et al., 2007).

Leonardi et al. (2010) evaluated both the effect isolated of $C$. curassavica essential oil (CV) and in association of glycolic acid $70 \%$ rats back skin. The association in base gel of glycolic acid (10\%) with CV 2\% applied once daily, for 15 consecutive days increased fibroblast counts without increasing leukocyte numbers, meaning inflammatory reaction may be reduced.

In addition to anti-inflammatory effect of the essential oil of C. curassavica, Passos et al. (2007), observed the anti-allergic effects. Systemic treatment with this essential oil (300-600 mg/kg, p.o.) and some active compounds $\alpha$-humulene and $\beta$-caryophyllene $(50 \mathrm{mg} / \mathrm{kg}$, p.o. $)$ - showed marked anti-inflammatory effects, probably by interfering with TNF alpha production. And, also indicated possible results for the anti-allergic action of the oil.

To verify biological activity antiperiodontitis, Ribeiro et al. (2012) evaluated the effect of crude extract and essential oil of $C$. curassavica, systemically administered, on ligatureinduced periodontitis in rats. Treatment with 100 $\mathrm{mg} / \mathrm{kg} /$ day orally 3 times a day for 11 days. In results both extract and essential oil orally administered may attenuate the progression of ligature induced periodontitis. The same group after, evaluated the effect of $C$. curassavica essential oil topically administered in a rat periodontitis model. The topical preparation of $C$. curassavica oil was also effective in periodontitis, concluding the possibility of mediation, in part, by its inhibition effect on periodontal pathogens and, in part, by their modulatory role in the immune-inflammatory response (Pimentel et al., 2012).

Nizio et al. (2017) evaluate the antiprotozoal activity of essential oils from $V$. curassavica accessions against different stages of Ichthyophthirius multifiliis. A significant reduction was observed in vivo assay from about $30 \%$ in the disease caused by the protozoan, independent of the oil concentration $(0.5,1.0,1.5$ and $2.0 \mathrm{mg} / \mathrm{L})$.

Carvalho \& Barja (2012) evaluated the penetration kinetics of $C$. curassavica essential oil in human skin, employing in vivo measurements after massage application or phonophoresis application. The measurements have shown drug penetration for both application forms, and the delivery was more evident after phonophoresis application.

Although antimicrobial activity has been the largest citation for biological activity of Cordia essential oils, no pre-clinical studies have been identified. All the works of analysis of this activity $(100 \%)$ were related to in vitro tests.

\section{Clinical evaluation}

Among the 60 studies, one of them (1.7\%) addressed the clinical evaluation of essential oil, from $C$. curassavica. The essential oil $(0.5 \%)$ under analysis was incorporated into a pharmaceutical dosage form (cream), and the efficacy and tolerability of the topical use in patients with myofascial pain and chronic tendonitis were evaluated in comparison with diclofenac diethylammonium $1 \%$. In results, the cream containing C. curassavica $0.5 \%$ essential oil was effective in the treatment of myofascial pain and chronic tendonitis in the local application of the lesion (every 8 hours). Compared to diclofenac diethylammonium $1 \%$ emulgel, the cream of $C$. curassavica $0.5 \%$ showed similar efficacy for cases of myofascial pain and superior in cases of chronic tendonitis. Regarding tolerability, both the essential oil cream of $C$. curassavica and diethylammonium diclofenac were considered, by the users, excellent in most cases (Refsio et al., 2005).

\section{DISCUSSION}

Even considering the difficulties in verifying the official name and synonymies, C. curassavica was the most cited name in the analyzed studies regarding essential oil. Citing botanical nomenclature not accepted or inconsistent with reference databases may lead to a study approach of one species erroneously. An incorrect citation may represent a difficulty for data processing in a review due to the difficulty in reporting the corresponding species.

C. curassavica is a plant native to Brazil with occurrence and distribution throughout large regions from the Americas, mainly from the South and Central (Rosa et al., 2008), and widely used through different forms, such as decoction, infusion, and alcoholic extracts (Medeiros et al., 2007). For this species, there are vast reports of traditional use, such as to treat rheumatism, rheumatoid arthritis, gout, muscle, and spinal pain, neuralgia, prostatitis, bruising, and as antiulcerogenic, antimicrobial, and tonic remedies (Panizza, 1997; Passos et al., 2007).

Compared to a literature review done by Thirupathi et al. (2008), the survey of biological activities described in this work resembles those 
already cited for the genus Cordia, such as antiinflammatory, antimicrobial, anti-allergic, antinociception, and larvicidal activities (Thirupathi et al., 2008). The antioxidant, acaricidal, bacterial resistance modulating, acetylcholinesterase inhibitory, butyryl cholinesterase inhibitory, antiperiodontitis, antiprotozoal, food deterrent and pesticidal activities were discussed for the first time in a systematic review work.

Among all the activities studied, the most prominent was the antimicrobial. It should be emphasized that, despite the existence of the studies on Cordia biological activity, further clinical trials of extracts, essential oils, or isolated compounds are needed, as well as toxicological tests in vitro and in vivo to ensure their safety, considering in reviewing the literature, only one study addressed clinical evaluation as the essential oil of a species of Cordia was found.

The works cited in this review that evaluated the antimicrobial activity (antibacterial and antifungal), all were related to laboratory analyses in vitro studies. Even being the activity with the highest citation regarding the biological action of Cordia essential oils, it was not possible to verify the efficacy and safety of the use with this approach. Therefore, a deficient gap in pre-clinical and clinical evaluations is perceived, suggesting further studies in these phases. This was different for the biological anti-inflammatory activity, which although not the most cited, the studies of this activity included both in vitro analysis and the results corroborated in preclinical and clinical evaluations (Refsio et al., 2005; Fernandes et al., 2007; Medeiros et al., 2007; Passos et al., 2007; Leonardi et al., 2010; Basting et al., 2019).

Of the 60 articles that approached the chemical compounds of the essential oil, the most commonly described compounds were $\beta$ caryophyllene (26\%), $\alpha$-humulene (13\%). $\alpha$ Humulene and $\beta$-caryophyllene are extracted by hydrodistillation process and they can be found in abundance in the essential oil extracted from the leaves of species of the genus Cordia. These compounds are the active ingredients found in the leaves of the $C$. curassavica and they are described as the main elements of the anti-inflammatory activity.

Some methodological limitations of this review should be considered. It should be noted that there is no quality standard assessment tool specific to the evaluated studies. Some nomenclature citations were not in accordance with the reference database, representing a problem to the data treatment. Therefore, we based the information in the database The World Flora correlating the accepted nomenclature and its synonyms.

\section{CONCLUSIONS}

This review gathers information about the genus Cordia, presents aspects of essential oil extraction, biological activity, and chemical composition. From the analysis of the study results, $C$. curassavica was identified as the most studied species. For essential oil extraction, leaves were of the most used plant part. Different biological activities have been reported, such as: antibacterial, antifungal, antiinflammatory, larvicidal, antioxidant, bacterial resistance modulating, antiprotozoal, acaricidal, antiallergic, antinociception, acetylcholinesterase inhibitory, butyrylcholinesterase inhibitory, antiperiodontitis, cytotoxicity, food deterrent e pesticidal. Of all the biological activities studied, antimicrobial was more related. Several types of compounds have been identified from essential oil Cordia, mainly secondary metabolites of the class of terpenoids and hydrocarbons. The major compounds reported in essential oils were $\beta$-caryophyllene, $\alpha$-humulene, $\alpha$ pinene, bicyclogermacrene, and sabinene. The information reported in this review can contribute scientifically to guide research towards a new perspective with Cordia essential oils.

\section{ACKNOWLEDGMENTS}

The authors sincerely thank the University of Brasilia (UnB) for providing support to perform the present research.

\section{REFERENCES}

Flora do Brasil. 2019. Cordia in Flora do Brasil 2020 em construção. Jardim Botânico do Rio de Janeiro. http://floradobrasil.jbrj.gov.br/reflora/floradobrasil/FB16502

Adeosun CB, Olaseinde S, Opeifa AO, Atolani O. 2013. Essential oil from the stem bark of Cordia sebestena scavenges free radicals. J Acute Med 3: 138 - 141. https://doi.org/10.1016/j.jacme.2013.07.002 
Adeosu CB, Bamigbade OL, Osho A, Atolani O. 2015. Volatile composition of the leaf, flower and fruit of Cordia sebestena (L.). J Essent Oil-Bear Plants 18: 976 - 981. https://doi.org/10.1080/0972060X.2014.884758

Alves MS, DP Santos, Silva LCP, Pontes EG, Souza MAA. 2015. Essential oils composition and toxicity tested by fumigation against Callosobruchus maculatus (Coleoptera: Bruchidae) pest of stored cowpea. Rev Virtual Quim 7: 2387 - 2399. https://doi.org/10.5935/1984-6835.20150142

Ambrosio CMS, Alencar SM, Sousa RLM, Moreno AM, Gloria EM. 2017. Antimicrobial activity of several essential oils on pathogenic and beneficial bacteria. Ind Crops Prod 97: 128 - 136. https://doi.org/10.1016/j.indcrop.2016.11.045

Arze JBL, Collin G, Garneau FX, Jean FI, Gagnon H. 2013. Essential oils from Bolivia. XI. Verbenacea: Aloysia gratissima (Gillies \& Hook.) Tronc. and Boraginaceae: Cordia chacoensis Chodat. J Essent Oil-Bear Plants 16: 545 - 550. https://doi.org/10.1080/0972060X.2013.831553

Ashmawy AM, Ayoub IM, Eldahshan OA. 2020. Chemical composition, cytotoxicity and molecular profiling of Cordia africana Lam. on human breast cancer cell line. Nat Prod Res 1 - 6. https://doi.org/10.1080/14786419.2020.1736064

Basting RT, Spindola HM, Sousa IMO, Queiroz NCA, Trigo JR, Carvalho JE, Foglio MA. 2019. Pterodon pubescens and Cordia verbenacea association promotes a synergistic response in antinociceptive model and improves the anti-inflammatory results in animal models. Biomed Pharmacother 112: 108693 108693. https://doi.org/10.1016/j.biopha.2019.108693

Bonesi M, Okusa PN, Tundis R, Loizzo MR, Menichini F, Stévigny C, Duez P, Menichini F. 2011. Chemical composition, antioxidant properties and anti-cholinesterase activity of Cordia gilletii (Boraginaceae) leaves essential oil. Nat Prod Commun 6: 253 - 257. https://doi.org/10.1177/1934578x1100600225

Carvalho PM, Rodrigues RF, Sawaya AC, Marques MO, Shimizu MT. 2004. Chemical composition and antimicrobial activity of the essential oil of Cordia verbenacea D.C. J Ethnopharmacol 95: 297 - 301. https://doi.org/10.1016/j.jep.2004.07.028

Carvalho SS, Barja PR. 2012. Photoacoustic analysis of the penetration kinetics of Cordia verbenacea DC in human skin. Int J Thermophys 33: 1822 - 1826. https://doi.org/10.1007/s10765-012-1230-0

Carvalho VRDA, Silva MKDN, Aguiar JJS, Bitu VDCN, Costa JGMD, Ribeiro-Filho J, Coutinho HDM, Pinho AI, Matias EFF. 2017. Antibiotic-modifying activity and chemical profile of the essential oil from the leaves of Cordia verbenacea DC. J Essent Oil-Bear Plants 20: 337 - 345.

https://doi.org/10.1080/0972060X.2017.1301220

Castro KNC, Costa-Júnior LM, Lima DF, Canuto KM, Brito ES, Andrade IM, Teodoro MS, Oiram-Filho F, Santos RC, Mayo SJ. 2019. Acaricidal activity of cashew nut shell liquid associated with essential oils from Cordia verbenacea and Psidium guajava on Rhipicephalus microplus. J Essential Oil Res 31: 297 - 304. https://doi.org/10.1080/10412905.2019.1580225

Chaves JS, Leal PC, Pianowisky L, Calixto JB. 2008. Pharmacokinetics and tissue distribution of the sesquiterpene alpha-humulene in mice. Planta Med 74: 1678 - 1683. https://doi.org/10.1055/s-0028-1088307

Diniz JC, Viana FA, Oliveira OF, Silveira ER, Pessoa ODL. 2008. Chemical composition of the leaf essential oil of Cordia leucocephala Moric from northeast of Brazil. J Essential Oil Res 20: 495 - 496. https://doi.org/10.1080/10412905.2008.9700068

Duarte MCT, Figueira GM, Sartoratto A, Rehder VLG, Delarmelina C. 2005. Anti-candida activity of Brazilian medicinal plants. J Ethnopharmacol 97: 305 - 311. https://doi.org/10.1016/j.jep.2004.11.016

Facanali R, Marques MOM, Hantao LW. 2020. Metabolic profiling of Varronia curassavica Jacq. terpenoids by flow modulated two-dimensional gas chromatography coupled to mass spectrometry. Separations 7. https://doi.org/10.3390/separations7010018

Feijó EVRS, Oliveira RA, Costa LCB. 2014. Light affects Varronia curassavica essential oil yield by increasing trichomes frequency. Rev Bras Farmacogn 24: 516 - 523. https://doi.org/10.1016/j.bjp.2014.10.005

Fernandes ES, Passos GF, Medeiros R, Cunha FM, Ferreira J, Campos MM, Pianowski LF, Calixto JB. 2007. Antiinflammatory effects of compounds alpha-humulene and (-)-trans-caryophyllene isolated from the essential oil of Cordia verbenacea. Eur J Pharmacol 569: 228 - 236. https://doi.org/10.1016/j.ejphar.2007.04.059

Fun CE, Svendsen AB. 1990. The essential oil of Cordia cylindrostachya Roem. \& Schult. grown on Aruba. J 
Essent Oil Res 2: 209 - 210. https://doi.org/10.1080/10412905.1990.9697864

Gomes MVS, Silva JD, Ribeiro AF, Cabral LM, Sousa VP. 2019. Development and validation of a quantification method for $\alpha$-humulene and trans-caryophyllene in Cordia verbenacea by high performance liquid chromatography. Rev Bras Farmacogn 29: 182 - 190. https://doi.org/10.1016/j.bjp.2019.01.009

Gómez NE, Witte L, Hartmann T. 1999. Chemical defense in larval tortoise beetles: essential oil composition of fecal shields of Eurypedus nigrosignata and foliage of its host plant, Cordia curassavica. J Chem Ecol 25: 1007 - 1027. https://doi.org/10.1023/A:1020821507014

Gracas M, Zoghbi B, Andrade EHA, Pereira RA, Oliveira J. 2010. Volatiles of the Cordia multispicata Cham.: A weed medicinal Brazilian plant. J Essential Oil Res 22: 543 - 545.

https://doi.org/10.1080/10412905.2010.9700395

Guimarães LGL, Cardoso MG, Lucas EMF, Freitas MP, Francisco W, Nelson DL. 2015. Structural elucidation of a new sesquiterpene alcohol by comparative NMR studies. Rec Nat Prod 9: 201 - 207.

Hernandez T, Canales M, Teran B, Avila O, Duran A, Garcia AM, Hernandez H, Angeles-Lopez O, FernandezAraiza M, Avila G. 2007. Antimicrobial activity of the essential oil and extracts of Cordia curassavica (Boraginaceae). J Ethnopharmacol 111: 137 - 141. https://doi.org/10.1016/j.jep.2006.11.002

Hernández D, Orozco J, Serrano R, Duran A, Meraz S, Jimenez-Estrada M, García-Bores A, Avila JG, Hernández T. 2014. Temporal variation of chemical composition and antimicrobial activity of the essential oil of Cordia curassavica (Jacq.) Roemer and Schultes: Boraginaceae. Bol Latinoam Caribe Plant Med Aromat 13: 100 - 108.

Hoyos JMA, Alves E, Rozwalka LC, Souza EA, Zeviani WM. 2012. Antifungal activity and ultrastructural alterations in Pseudocercospora griseola treated with essential oils. Cienc Agrotecn 36: 270 - 284. https://doi.org/10.1590/S1413-70542012000300002

Kendir G, Özek G, Özek T, Köroğlu A. 2020. Leaf essential oil analysis and anatomical study of Cordia myxa from Turkey. Plant Biosyst 26: 204 - 210. https://doi.org/10.1080/11263504.2020.1762788

Kumar V, Shriram V, Bhagat R, Khare T, Kapse S, Kadoo N. 2019. Phytochemical profile, anti-oxidant, antiinflammatory, and anti-proliferative activities of Pogostemon deccanensis essential oils. 3 Biotech 9: 1 12. https://doi.org/10.1007/s13205-018-1560-0

Kurti F, Giorgi A, Beretta G, Mustafa B, Gelmini F, Testa C, Angioletti S, Giupponi L, Zilio E, Pentimalli D, Hajdari A. 2019. Chemical composition, antioxidant and antimicrobial activities of essential oils of different Pinus species from Kosovo. J Essential Oil Res 31: 263 - 275.

https://doi.org/10.1080/10412905.2019.1584591

Lasram S, Zemni H, Hamdi Z, Chenenaoui S, Houissa H, Tounsi MS, Ghorbel A. 2019. Antifungal and antiaflatoxinogenic activities of Carum carvi L., Coriandrum sativum L. seed essential oils and their major terpene component against Aspergillus flavus. Ind CropsProd 134: 11 - 18.

https://doi.org/10.1016/j.indcrop.2019.03.037

Leonardi GR, Rigon RB, Cavallini ME, Polacow MLO, Araújo MR, Andreollo NA. 2010. Topical formulation with Cordia verbenacea essential oil and glycolic acid. Latin Am J Pharm 29: 1004 - 1008.

Marques APS, Bonfim FPG, Dantas WFC, Puppi RJ, Marques MOM. 2019. Chemical composition of essential oil from Varronia curassavica Jacq. accessions in different seasons of the year. Ind Crops Prod 140: 111656. https://doi.org/10.1016/j.indcrop.2019.111656

Matias EFF, Alves EF, Silva MKN, Carvalho VRA, Coutinho HDM, Costa JGM. 2015. The genus Cordia: botanists, ethno, chemical and pharmacological aspects. Rev Bras Farmacogn 25: 542 - 552. https://doi.org/10.1016/j.bjp.2015.05.012

Matias EFF, Alves EF, Silva MKN, Carvalho VRA, Figueredo FG, Ferreira JVA, Coutinho HDM, Silva JMLF, Ribeiro-Filho J, Costa JGM. 2016. Seasonal variation, chemical composition and biological activity of the essential oil of Cordia verbenacea DC (Boraginaceae) and the sabinene. Ind Crops Prod 87: 45 - 53. https://doi.org/10.1016/j.indcrop.2016.04.028

Meccia G, Rojas LB, Velasco J, Díaz T, Usubillaga A, Arzola JC, Ramos S. 2009. Chemical composition and antibacterial activity of the essential oil of Cordia verbenacea from the Venezuelan Andes. Nat Prod Commun 4: 1119 - 1122. https://doi.org/10.1177/1934578x0900400821

Boletín Latinoamericano y del Caribe de Plantas Medicinales y Aromáticas / 172 
Medeiros R, Passos GF, Vitor CE, Koepp J, Mazzuco TL, Pianowski LF, Campos MM, Calixto JB. 2007. Effect of two active compounds obtained from the essential oil of Cordia verbenacea on the acute inflammatory responses elicited by LPS in the rat paw. Br J Pharmacol 151: 618 - 627.

https://doi.org/10.1038/sj.bjp.0707270

Menezes JESA, Lemos TLG, Silveira ER, Pessoa ODL, Santiago GMP, Nascimento RF. 2006. Chemical composition and larvicidal activity of the essential oil from leaves of Cordia globosa (Jacq.) H.B.K. from Northeastern Brazil. J Essential Oil Res 18: 253 - 255. https://doi.org/10.1080/10412905.2006.9699079

Menezes JESA, Lemos TLG, Silveira ER, Andrade-Neto M, Nascimento RF, Pessoa ODL. 2005. Volatile constituents of Cordia trichotoma Vell. from the northeast of Brazil. Flavour Fragr J 20: 149 - 151. https://doi.org/10.1002/ffj.1394

Michielin EMZ, Salvador AA, Riehl CAS, Smânia A, Smânia EFA, Ferreira SRS. 2009. Chemical composition and antibacterial activity of Cordia verbenacea extracts obtained by different methods. Biores Technol 100: 6615 - 6623. https://doi.org/10.1016/j.biortech.2009.07.061

Miller RW, Earle FR, Wolff IA, Barclay AS. 1968. Search for new seed oils. XV. Oils of Boraginaceae. Lipids 3: 43 - 45. https://doi.org/10.1007/bf02530967

Moher D, Liberati A, Tetzlaff J, Altman DG. 2009. Preferred reporting items for systematic reviews and metaanalyses: the PRISMA statement. Plos Med 6: e1000097. https://doi.org/10.1371/journal.pmed.1000097

Nizio DAC, Brito FA, Sampaio TS, Melo JO, Silva FLS, Gagliardi PR, Arrigoni-Blank MF, Anjos CS, Alves PB, Wisniewski Junior A, Blank AF. 2015. Chemical diversity of native populations of Varronia curassavica Jacq. and antifungal activity against Lasiodoplodia theobromae. Ind Crops Prod 76: 437 - 448. https://doi.org/10.1016/j.indcrop.2015.07.026

Nizio DAC, Fujimoto RY, Maria AN, Carneiro PCF, França CCS, Sousa NC, Brito FA, Sampaio TS, ArrigoniBlank MF, Blank AF. 2018. Essential oils of Varronia curassavica accessions have different activity against white spot disease in freshwater fish. Parasitol Res 117: 97 - 105.

https://doi.org/10.1007/s00436-017-5673-x

Oliveira JCS, Camara CAG, Schwartz MOE. 2007. Volatile constituents of the stem and leaves of cordia species from mountain forests of Pernambuco (north-eastern Brazil). J Essential Oil Res 19: 444 - 448. https://doi.org/10.1080/10412905.2007.9699947

Panizza S. 1997. Plantas que curam (cheiro de mato). IBRASA, São Paulo, Brasil.

Passos GF, Fernandes ES, Cunha FM, Ferreira J, Pianowski LF, Campos MM, Calixto JB. 2007. Antiinflammatory and anti-allergic properties of the essential oil and active compounds from Cordia verbenacea. J Ethnopharmacol 110: 323 - 333. https://doi.org/10.1016/j.jep.2006.09.032

Perini JA, Angeli-Gamba T, Alessandra-Perini J, Ferreira LC, Nasciutti LE, Machado DE. 2015. Topical application of Acheflan on rat skin injury accelerates wound healing: a histopathological, immunohistochemical and biochemical study. BMC Complement Altern Med 15: 203 - 203. https://doi.org/10.1186/s12906-015-0745-x

Pimentel SP, Barrella GE, Casarin RC, Cirano FR, Casati MZ, Foglio MA, Figueira GM, Ribeiro FV. 2012. Protective effect of topical Cordia verbenacea in a rat periodontitis model: immune-inflammatory, antibacterial and morphometric assays. BMC Complement Altern Med 12: 224. https://doi.org/10.1186/1472-6882-12-224

Pino JA, Bello A, Urquiola A, Marbot R. 2002. Fruit volatiles from Cordia nitida Vahl. J Essential Oil Res 14: 118 - 119. https://doi.org/10.1080/10412905.2002.9699790

Queiroz TB, Mendes ADR, Silva JCRL, Fonseca FSA, Martins ER. 2016. Teor e composição química do óleo essencial de erva-baleeira (Varronia curassavica Jaqc.) em função dos horários de coleta. Rev Bras Plantas Med 18: 356 - 362. https://doi.org/10.1590/1983-084x/15_116

Quispe-Condori S, Foglio MA, Rosa PTV, Meireles MAA. 2008. Obtaining $\beta$-caryophyllene from Cordia verbenacea de Candolle by supercritical fluid extraction. J Supercritical Fluids 46: 27 - 32. https://doi.org/10.1016/j.supflu.2008.02.015

Rassem HHA, Nour AH, Yunus RM. 2016. Techniques for extraction of essential oils from plants: a review. Australian J Basic Applied Sci 10: 117 - 127.

Boletín Latinoamericano y del Caribe de Plantas Medicinales y Aromáticas / 173 
Refsio C, Brandão DC, Korukian M, Garcia RJ, Bonfiglioli R. 2005. Avaliação clínica da eficácia e segurança do uso de extrato padronizado da Cordia verbenacea em pacientes portadores de tendinite e dor miofascial. Rev Bras Med 62: 40 - 46. https://doi.org/10.11606/d.10.2006.tde-11072007-140524

Ribeiro FV, Barrella GE, Casarin RCV, Cirano FR, Foglio MA, Pimentel SP. 2012. Effect of crude extract and essential oil of Cordia verbenacea in experimental periodontitis in rats. Braz J Oral Sci 11: 42 - 46. https://doi.org/10.1186/1472-6882-12-224

Rodrigues FF, Oliveira LG, Rodrigues FF, Saraiva ME, Almeida SC, Cabral ME, Campos AR, Costa JG. 2012. Chemical composition, antibacterial and antifungal activities of essential oil from Cordia verbenacea DC leaves. Pharmacogn Res 4: 161 - 165. https://doi.org/10.4103/0974-8490.99080

Rosa DD, Basseto MA, Feliciano F, Neves MB, Baldin ELL. 2008. Ocorrência de Dictyla monotropidia Stål (Hemiptera: Tingidae) em Cordia verbenacea Al. DC no Brasil. Neotrop Entomol 37: 236 - 238. https://doi.org/10.1590/s1519-566x2008000200021

Saldanha AA, Vieira L, Ribeiro RIMA, Thomé RG, Santos HB, Silva DB, Carollo CA, Oliveira FM, Lopes DO, Siqueira JM, Soares AC. 2019. Chemical composition and evaluation of the anti-inflammatory and antinociceptive activities of Duguetia furfuracea essential oil: Effect on edema, leukocyte recruitment, tumor necrosis factor alpha production, iNOS expression, and adenosinergic and opioidergic systems. J Ethnopharmacol 231: 325 - 336. https://doi.org/10.1016/j.jep.2018.11.017

Sánchez DV, Galvão JA, Ambrosio CMS, Gloria EM, Oetterer M. 2018. Single and binary applications of essential oils effectively control Listeria monocytogenes biofilms. Ind Crops Prod 121: 452 - 460. https://doi.org/10.1016/j.indcrop.2018.05.045

Santos RP, Nunes EP, Nascimento RF, Santiago GMP, Menezes GHA, Silveira ER, Pessoa ODL. 2006. Chemical composition and larvicidal activity of the essential oils of Cordia leucomalloides and Cordia curassavica from the Northeast of Brazil. J Braz Chem Soc 17: 1027 - 1030. https://doi.org/10.1590/S0103-50532006000500030

Santos AV, Antunes e Defaveri AC, Bizzo HR, Aguiar da Silva San Gil R, Sato A. 2013. In vitro propagation, histochemistry, and analysis of essential oil from conventionally propagated and in vitro-propagated plants of Varronia curassavica Jacq. In Vitro Cell Develop Biol Plant 49: 405 - 413. https://doi.org/10.1007/s11627-013-9528-6

Sciarrone D, Giuffrida D, Rotondo A, Micalizzi G, Zoccali M, Pantò S, Donato P, Rodrigues-das-Dores RG, Mondello L. 2017. Quali-quantitative characterization of the volatile constituents in Cordia verbenacea D.C. essential oil exploiting advanced chromatographic approaches and nuclear magnetic resonance analysis. J Chromatogr A 1524: 246 - 253. https://doi.org/10.1016/j.chroma.2017.10.007

Silva AC, Souza PE, Amaral DC, Zeviani WM, Pinto JEBP. 2014a. Essential oils from Hyptis marrubioides, Aloysia gratissima and Cordia verbenacea reduce the progress of Asian soybean rust. Acta Scient Agron 36: 159 - 166. https://doi.org/10.4025/actasciagron.v36i2.17441

Silva AC, Souza PE, Machado JC, Silva BM, Pinto JEBP. 2012a. Effectiveness of essential oils in the treatment of Colletotrichum truncatum-infected soybean seeds. Trop Plant Pathol 37: 305 - 313. https://doi.org/10.1590/S1982-56762012000500001

Silva AC, Souza PE, Pinto JEBP, Silva BM, Amaral DC, Carvalho EA. 2012b. Essential oils for preventative treatment and control of Asian soybean rust. Eur J Plant Pathol 134: 865 - 871. https://doi.org/10.1007/s10658-012-9962-z

Silva AC, Souza PE, Resende MLV, Silva MB, Ribeiro PM, Zeviani WM. 2014b. Local and systemic control of powdery mildew in eucalyptus using essential oils and decoctions from traditional Brazilian medicinal plants. Forest Pathol 44: 145 - 153. https://doi.org/10.1111/efp.12079

Silva JD, Gomes MV, Cabral LM, Sousa VP. 2019b. Evaluation of the in vitro release and permeation of Cordia verbenacea DC essential oil from topical dosage forms. J Drug Delivery Sci Technol 53: 101173. https://doi.org/10.1016/j.jddst.2019.101173

Silva KP, Santos TAC, Moutinho BL, Silva RS, Pinto VS, AF Blank, Corrêa CB, Scher R, Fernandes RPM. 2019a. Using Varronia curassavica (Cordiaceae) essential oil for the biocontrol of Phytomonas serpens. Ind Crops Prod 139: 111523. https://doi.org/10.1016/j.indcrop.2019.111523

Boletín Latinoamericano y del Caribe de Plantas Medicinales y Aromáticas / 174 
Silva RS, Oliveira MMG, Silva KP, Rodrigues ISV, Pinto VS, Blank AF, Fernandes RPM. 2019c. Synergistic effect of Cordia curassavica Jacq. essential oils association against the phytopathogen Xanthomonas campestris pv. campestris. Environ Sci Pollution Res 27: 4376 - 4389. https://doi.org/10.1007/s11356019-06631-8

Thirupathi K, Kumar SS, Raju VS, Ravikumar B, Krishna DR, Mohan GK. 2008. A review of medicinal plants of the genus Cordia: Their chemistry and pharmacological uses. J Nat Remedies 8: 1-10.

Ventrella MC, Marinho CR. 2008. Morphology and histochemistry of glandular trichomes of Cordia verbenacea DC. (Boraginaceae) leaves. Rev Bras Bot 31: 457 - 467. https://doi.org/10.1590/S0100-84042008000300010

Wei C, Zhou S, Li W, Jiang C, Yang W, Han C, Zhang C, Shao H. 2019. Chemical composition and allelopathic, phytotoxic and pesticidal activities of Atriplex cana Ledeb. (Amaranthaceae) essential oil. Chem Biodiv 16: 1 - 8. https://doi.org/10.1002/cbdv.201800595

WFL. 2020. World Flora online, World Flora Consortium. http://www.worldfloraonline.org

Wille VKD, Wastowski AD, Pedrazzi C, Sauer MP. 2017. Chemical composition of Cordia trichotoma (Vell.) Arráb. ex steud wood. Ciencia Florestal 27: 1441 - 1449. https://doi.org/10.5902/1980509830332

Zotti-Sperotto NC, Melo EC, Souza MIL, Fonseca MCM, Gonzaga DA, Ávila MBR, Demuner AJ, Ventrella MC, Lelis ACV. 2020. Effect of drying with ultrasonic pretreatment on the yield and quality of the essential oil of Varronia curassavica Jacq. and Ocimum gratissimum Linn. Ind Crops Prod 147: 112211.

https://doi.org/10.1016/j.indcrop.2020.112211 